\section{Statistical Optics}

Joseph W. Goodman, xvii +550 pp., illus., index, references, appendices. ISBN 0-471-01502-4. John Wiley \& Sons, Inc., 605 Third Ave., New York, N.Y. 10016 (1985) \$44.95 hardbound

Reviewed by Leonard Mandel, University of Rochester, Dept. of Physics and Astronomy, Rochester, N.Y. 14627.

Optics is fundamentally a statistical science, although this is not usually apparent in the traditional treatments. Statistics enters at three different levels, even when the optical field is treated classically: (a) The medium through which the light propagates may fluctuate. An obvious example is the atmosphere. (b) The optical field, being produced by atomic or molecular sources, undergoes fluctuations of amplitude and phase, which are manifest in various kinds of interference and correlation measurements. (c) There are fluctuations associated with the detection process itself, because the same optical field does not always elicit the same response from the same detector.

The latest book by Professor Goodman deals with all three of these different fluctuations and is an excellent exposition of classical statistical optics. The book is both an authoritative monograph and a graduate textbook, with each chapter followed by a number of exercise problems. The book starts with a good general introduction to probability and the theory of partial coherence, including the properties of optical correlation functions. The effects of coherence on image formation are then discussed for various kinds of sources. There is a long chapter on wave propagation through fluctuating media that includes the subject of speckle interferometry. The final chapter deals with the subject of photoelectric detection of light, including counting statistics and intensity interferometry. The electromagnetic field is treated classically throughout the book, which ends with three useful appendices on the Fourier transform, on random phasor sums, and on some moment calculations in speckle interferometry. Each chapter is followed by an extensive set of references, and the book has a good subject index.

The most attractive features of the book are the clear expository writing and the large number of illustrative figures in what is basically a mathematical text. It is apparent that the author has consulted the original literature in many cases, and he has obviously taken trouble to make his presentation clear, based on his considerable teaching experience. Moreover, he has pulled together results from many different sources that cannot normally be found between the same covers. The chapters on imaging with partially coherent light and in fluctuating media are particularly successful.

There are a few things that this reviewer would prefer had been done differently. The terms first order and higher order correlations are used here in the sense of one-time and multitime correlations and do not refer to the order of the field amplitude or of the light intensity, as they normally do. It would have been advantageous to define correlation functions in terms of ensemble averages rather than time averages. Although it makes no difference for an ergodic process, when nonergodic processes are considered there is often no such thing as the time average; indeed, there may be an infinity of different time averages. But even when multiple averages are not a problem, the ensemble average often has advantages. For example, some long time limits, such as that in Eq. (4.1-4), may not exist, and the paradox referred to on p. 134 and in Prob. 4-6 goes away if ensemble averages are used instead of time averages. As the author points out in Secs. 5.4.2 and 5.4.3, the cross-spectral density obeys the Helmholtz equation exactly, but the mutual intensity in general does not, and Eqs. (5.4-19) do not have the same degree of validity as Eqs. (5.4-22). The statement on p. 465 that the predictions of semiclassical theory coincide with those of the quantum theory for all problems involving the photoelectric effect needs the qualifier "for all states that are describable classically." There are a few effects, like subPoissonian photoelectric counting statistics, that are not described by the semiclassical theory.

None of these remarks alters the fact that this is the best all-round book on classical statistical optics. It is a very attractive introductory textbook for a graduate course. It contains a great deal of well-organized information, and it is likely to become the standard reference for workers in the field. As book prices go these days, even a $\$ 44.95$ price tag must be regarded as moderate.

\section{Free-Electron Lasers}

Thomas C. Marshall, xii +191 pp., illus., index, references. ISBN 0-02-948620-3. Macmillan Publishing Company, 866 Third Ave., New York, N.Y. 10022 (1985) \$24.95 hardbound.

Reviewed by S. H. Gold, Naval Research Laboratory, Plasma Physics Division, Washington, D.C. 20375.

The field of free-electron laser (FEL) research has rapidly advanced over the last decade from one of early theoretical promise, combined with a few proof-of-principle experiments, to a field in which the theory is mature and the experiments are coming on-line at a rapidly accelerating pace. Thomas C. Marshall, a professor of applied physics at Columbia University, has written the first book devoted solely to FELs that is neither a conference proceedings nor a compendium of technical papers. This book is written as an introductory textbook on FELs, covering the historical development of the field, its present status, and its future potential. It includes all of the key theoretical concepts of FEL physics, combined with a discussion of the principal experimental developments.

The first task of any book on free-electron lasers is defining its subject. While the usual meaning of the acronym "laser" is well-defined, the term "free-electron laser" has a less clear-cut delineation. Marshall defines it as a "device which amplifies short-wavelength radiation by stimulated emission, using a beam of relativistic electrons," and he suggests a long-wavelength limit of approx- imately $1 \mathrm{~mm}$. Many in the FEL community would apply either broader or narrower limits to the term. Some restrict it solely to optical devices operating via the "usual" FEL interaction. This involves the production of radiation due to axial bunching in a relativistic electron beam caused by the ponderomotive potential created by the superposition of undulator and radiation fields. Others extend it to include longer-wavelength devices operating at millimeter and centimeter wavelengths. Despite the upper wavelength limit suggested by Marshall, this book deals thoroughly with the experimental results at short and long wavelengths and, additionally, deals briefly with related devices such as Cerenkov FELs and cyclotron autoresonance masers, thus in effect taking the broadest view of the meaning of the title.

The first chapter of this book is a general introduction to FEL history, technology, and applications. It is followed by a chapter entitled "Conceptual Background," which presents an elementary explanation of some of the fundamental FEL physical concepts. The topics include the character of electron motion in an undulator magnetic field, the origin of the relativistic Doppler contraction of the undulator wavelength to produce the much shorter wavelength FEL radiation, the concept of stimulated emission of radiation from a relativistic electron beam, and the effects of beam space charge on the nature of the FEL interaction. This chapter lays the groundwork for the more detailed theoretical development contained in subsequent chapters.

Chapters 3 and 4 emphasize the conceptual development of FEL physics. There is generally less concern for rigorous mathematical derivation than for the provision of sufficient mathematical detail to show the origin of important concepts. Chapter 3 treats the low current "two-wave" regime typical of IR and visible FEL devices using high voltage linear accelerators and storage rings, and shows the origin of FEL gain and saturation. It also covers efficiency enhancement via undulator tapering, gain enhancement via optical klystrons, and transient effects such as oscillation start-up and the problem of "laser lethargy." Chapter 4 treats the high current "three-wave" regime of the long-wavelength FEL devices using pulse-line accelerators and induction linacs, in which the interaction proceeds via a parametric coupling to a negative energy space-charge wave. A particularly valuable feature of these chapters is the demonstration of a self-consistent physical picture that shows the connection between these two very different regimes of operation and demonstrates the common physics, such as the ponderomotive potential and the generalized pendulum equation, underlying both.

Chapter 5 discusses different types of undulators and the character of the associated electron trajectories. It also treats the sources of inhomogeneous broadening of the FEL emission. Chapter 6 briefly presents a variety of devices that are included in a broad definition of the term FEL, but involve somewhat different physics, including the Cerenkov FEL and the cyclotron autoresonance maser. Some of these devices are potential competitors for the usual FEL at long wavelengths.

Continued on Page SR-120 


\section{SCHOTT ... Precision Optical Glass Made in America and More!!!}

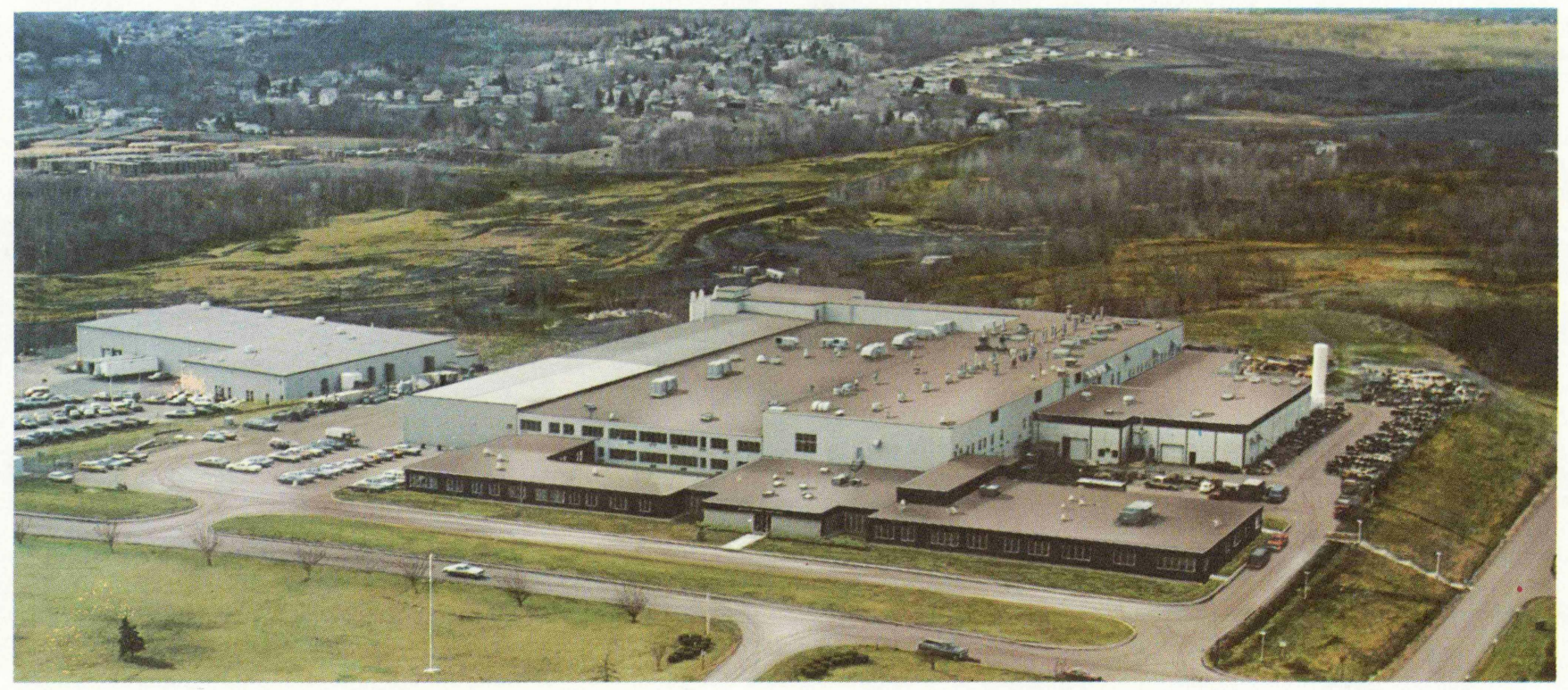

Schott Glass Technologies is geared to work with the largest production quantities or the smallest prototype development right here in the USA. Our 300,000 square feet of manufacturing facilities, backed by our scientific knowledge and technical skills, are ready to work for you at every stage of your program.

Our in-house research and development is among the most modern of its kind

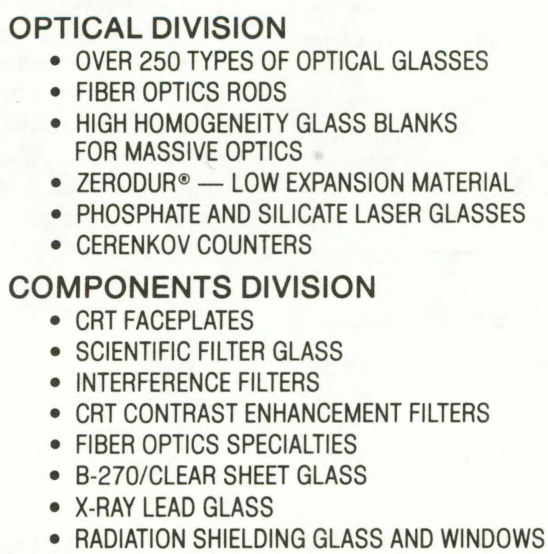

anywhere. And we have the marketing and sales know-how to assure you that products made from Schott materials or components get to your customers on time and to specification.

And, above all, Schott Glass Technologies has the widest range of precision optical glass and components available in the Western Hemisphere today.

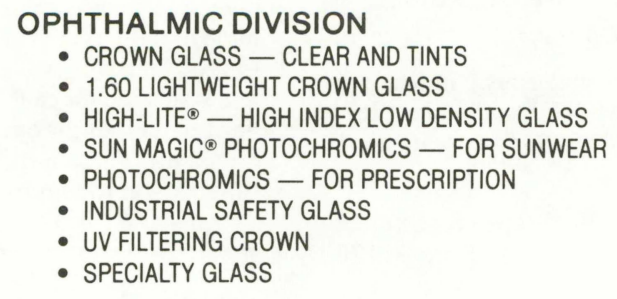

\section{TECHNICAL SERVICES}

- CONTRACT RESEARCH AND DEVELOPMENT

- CUSTOM MELTING

- ANALYTICAL/PHYSICAL/OPTICAL MEASUREMENTS

- and the list goes on ...

\section{Our commitment ... .}

\section{Research \& Development, Manufacturing, Sales, Service - Total Capability ... your advantage.}

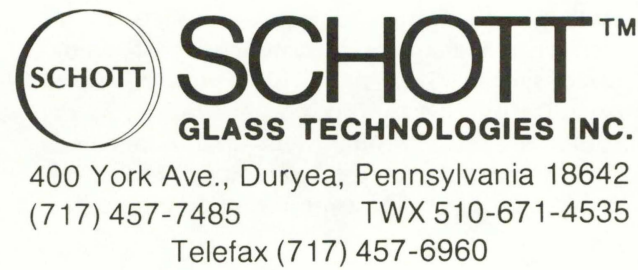




\section{Continued from Page SR-118}

The concluding two chapters describe in some detail the significant experimental results that have advanced the field to its present highly optimistic state. Separate chapters treat long- and short-wavelength experiments. Each chapter describes in detail the technology involved in the various types of accelerators that are used to generate FEL electron beams and the variety of experiments that have been carried out using such beams. The key results and their significance in confirming theoretical predictions and advancing the state of the art are covered in some detail. The coverage of the long-wavelength research is particularly thorough, evidencing the author's long association with this field of experimental research.

The specialist in FEL physics has already had a large assortment of conference proceedings and review articles to supplement the technical literature. Nevertheless, Free-Electron Lasers will likely be found on the shelf of most serious FEL researchers, since it provides a useful, cohesive viewpoint on the development of the field and of its state as of 1985 . However, there has been no introductory treatment of the field available for the nonspecialists and for those just entering the field. Marshall's book should fill this previous vacuum. While it is not an engineering text and will not tell how to build an FEL, it will tell why one might want to and explain its potential operating characteristics. Particularly valuable is the extensive bibliography of technical literature that accompanies the text, so that each topic may be pursued in more detail than is appropriate for an introductory textbook. This book belongs on the shelf of those who may be future users of FEL research facilities and should be interesting reading to those with a background in conventional "quantum mechanical" lasers who desire an understanding of their more "classical" new competitor

\section{Laser Dyes: Properties of Organic Compounds for Dye Lasers}

Mitsuo Maeda, x + 335 pp., illus., index, references. ISBN 0-12-464980-7. Academic Press, Inc., Orlando, Fla. 32887 (1984) $\$ 46.50$ hardbound, $£ 33.00$.

Reviewed by Richard N. Steppel, Exciton Chemical Company, Inc., P.O. Box 31126, Dayton, Ohio 45431.

This book was originally published in Japanese in Laser Kenkyu (The Review of Laser Engineering). For its present form, the text was revised and translated into English while maintaining the same dye and reference code numbers as in the original publication.

Although the book's six chapters make up more than 335 pages, the verbiage is definitely kept to a minimum. Of the 16 pages that make up the first three chapters, over six consist of figures and tables. The first chapter presents a brief historical review and delineates the nature of this publication: that is, this volume (1) contains information about 546 laser dyes compiled up to 1980 and is considered by the author to be the largest single compilation, (2) uses and correlates various types of chemical nomenclature as well as providing the exact structural composition for most of the dyes, and (3) cites many examples that provide experimental details with regard to the source of excitation and physical response of the dye in terms of laser output. The second chapter relates to the general definition, characterization, and classification of lasing compounds. The lasing materials are placed into three major categories, that is, dyes (Chap. 4), fluorescent aromatic compounds (Chap. 5), and fluorescent heterocyclic compounds (Chap. 6). Chapter 3 outlines the effect of the excitation source and the chemical and physical influences on the wavelength of lasing.

Chapters 4, 5, and 6, each consisting of tabulated data with a short introduction, form the heart of this compilation. These three chapters in conjunction with the two indices, a wavelength index and a compound index, allow the user to search for laser dyes according to either the wavelength region of choice or a specific dye name.

The wavelength index requires no specific knowledge of chemical nomenclature and is fairly easy to use when looking for the specified dye identified by its serial code number (\#M). This index covers the spectral region from $308.5 \mathrm{~nm}$ (\#M208) to $1285 \mathrm{~nm}$ (\#M70). However, since the $M$ numbers are not in bold face or conspicuously placed in the tables, they are sometimes difficult to find. The wavelength index is subdivided into 30 wavelength regions, consisting of $25 \mathrm{~nm}$ wavelength increments from $300 \mathrm{~nm}$ to $800 \mathrm{~nm}$ and $50 \mathrm{~nm}$ increments from $800 \mathrm{~nm}$ to $1300 \mathrm{~nm}$. This index will certainly be the more widely used by the dye laser user.

For those already having some knowledge of or wishing to become more familiar with the chemical nomenclature of certain laser dyes, one may try using the common or trivial name in the compound index or the more exacting chemical name, many of which have been included. For those who believe a picture is worth a thousand words, the author has included the chemical structure for almost all of the laser dyes and has been astute enough to recognize certain fallacies in the literature, such as the fact that the dyes Carbostyril 124 and Carbostyril 165 are not the same as LD 423 and LD 425 , as has been projected by others.

Thus, the heart of this compendium on laser dyes lies in the tables, which take up the vast majority of pages in this book. In the tables can be found the lasing wavelength, mode of excitation, and reference for each dye. In many cases the exact parameters for the excitation and resulting output are given. These latter values make this compilation different from its predecessors.

\section{Ion Implantation and Beam Processing}

J. S. Williams and J. M. Poate, eds., xi +419 pp., illus., index, references. ISBN 0-12-756980-4. Academic Press, Centrecourt, 25-26 Paul Street North, North Ryde, NSW 2113, Australia (1984) $\$ 57.50$ hardbound, $£ 41.00$.

Reviewed by Charter D. Stinespring, Aerodyne Research, Inc., Center for Chemical and Environmental Physics, Billerica, Mass. 01821.

Motivated by an international workshop held at Philip Island, Australia, in 1981, Ion Implantation and Beam Processing describes the impact of two allied technologies - ion implantation and ultrarapid heating using lasers-on electronic materials processing. The book consists of 11 well-written chapters contributed by 15 recognized specialists in the field. Through careful organization by the editors and authors, the reader is guided through both the fundamentals and applications of the technology. Moreover, because of the emphasis on recent developments, both the student and the specialized researcher will find this book of great interest.

Chapter 1 provides an introductory overview of fundamental processes including amorphization that are associated with implantation. This chapter also stresses the critical importance of recrystalization procedures to the ultimate success of implantation technologies. Chapters 2 through 8 expand on these fundamentals, while Chaps. 9 through 11 discuss their applications to specific semiconductor technologies.

Chapter 2 provides a more detailed overview of implantation-induced damage and amorphization and discusses the use of crystallization processes that employ pulsed lasers. In addition, advances in solid and liquid phase crystallization studies are overviewed.

Chapter 3 discusses the use of the Boltzmann transport equation to describe implant and damage distributions in the linear cascade regime. Chapter 4, on the other hand, discusses "spike" effects and their use to describe damage in the high-density cascades. Although well written, these chapters could easily have been expanded. Chapter 5 deals with the implantation phenomena that occur in insulators. Here the situation may be somewhat similar to that described in Chap. 4 since the deposited energy remains localized around the ion track, causing thermal or ionization spikes.

Chapters 6 and 7 provide an extensive discussion of ion-bombardment-induced composition changes. Chapter 6 overviews both collisional and chemical effects associated with ion sputtering. In particular, this chapter provides a good discussion of preferential sputtering and its relationship to thermal and radiation induced segregation and diffusion (i.e., chemical effects). Chapter 7 complements this discussion with an equally detailed overview of ion beam (as well as laser) mixing.

Chapter 8 discusses current and future applications of high-dose ion implantation, the effects of implant temperature on damage, and the behavior of high-dose implants. In addition, the hardware requirements for high-dose implants are discussed.

Chapters 9 through 11 describe the role of ion implantation and semiconductor device technology. Chapter 9 is devoted to silicon, while Chapter 10 is concerned with gallium arsenide. Chapter 11 describes the application of implantation technologies to the interconnect and contact technology for both silicon and gallium arsenide.

In summary, this book is well written and organized. It covers the subject material from fundamental concepts through to device applications and is well documented in terms of illustrations and references. This book is well worth reading, and I would recommend it to students and researchers alike.

\section{BOOKS RECEIVED}

Self-Study Manual on Optical Radiation Measurements, NBS Technical Note 910-6. Edited by Fred E. Nicodemus; issued March 1983. Available from Superintendent of Documents, U.S. Government Printing Office, Washington, DC 20402. Soft cover, $\$ 5,60$ pages.

Hot-Electron Transport in Semiconductors, edited by L. Reggiani. Springer-Verlag Publishing, 1985, 275 pages with index. Intended for graduate students and researchers. Sections include general theory, transport parameters, multivalued distributions, and non-steady-state transport. Hard cover, $\$ 42$. 